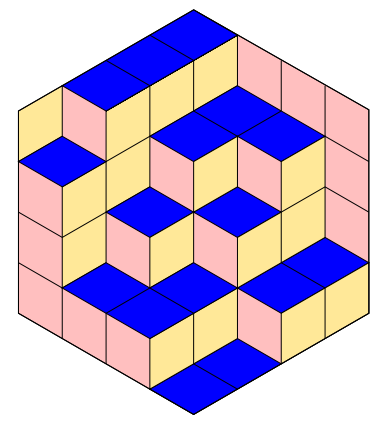

ALGEBRAIC COMBINATORICS

Jean-Christophe Novelli, Jean-Yves Thibon \& Frédéric Toumazet

Noncommutative Bell polynomials and the dual immaculate basis

Volume 1, issue 5 (2018), p. 653-676.

<http://alco.centre-mersenne.org/item/ALCO_2018__1_5_653_0>

(c) The journal and the authors, 2018.

Some rights reserved.

(c) BY This article is licensed under the

Creative Commons AtTribution 4.0 International License.

http://creativecommons.org/licenses/by/4.0/

Access to articles published by the journal Algebraic Combinatorics on the website http://alco.centre-mersenne.org/ implies agreement with the Terms of Use (http://alco.centre-mersenne.org/legal/).

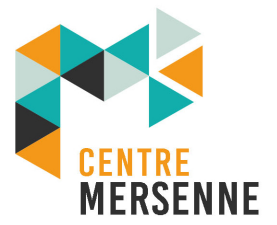

Algebraic Combinatorics is member of the Centre Mersenne for Open Scientific Publishing www.centre-mersenne.org 


\title{
Noncommutative Bell polynomials and the dual immaculate basis
}

\author{
Jean-Christophe Novelli, Jean-Yves Thibon \& Frédéric
}

Toumazet

\begin{abstract}
We define a new family of noncommutative Bell polynomials in the algebra of free quasi-symmetric functions and relate it to the dual immaculate basis of quasi-symmetric functions. We obtain noncommutative versions of Grinberg's results [14], and interpret these in terms of the tridendriform structure of WQSym. We then present a variant of Rey's self-dual Hopf algebra of set partitions [35] adapted to our noncommutative Bell polynomials and give a complete description of the Bell equivalence classes as linear extensions of explicit posets.
\end{abstract}

\section{INTRODUCTION}

Many classical combinatorial numbers or polynomials are the dimensions (or graded dimensions) of certain representations of the symmetric groups, and can therefore be regarded as specializations of the symmetric functions encoding the characters of these representations [7]. Classical examples include the Euler numbers [10], or the Eulerian polynomials [11]. In both cases, the generating series of the relevant symmetric functions can be obtained as the homomorphic images of multiplicity-free series living in some (in general noncommutative) combinatorial Hopf algebra. For example, the generating series $\tan x+\sec x$ of the Euler numbers is the image of the formal sum of all alternating permutations in the Hopf algebra of free quasisymmetric functions FQSym by its canonical character, and its lift by Foulkes to symmetric functions is just the commutative image of the same series [12, 19].

Other examples of this situation include the derangement numbers [15], the numbers of parking functions [29], the Abel polynomials [29] or Arnold's snakes [19].

These combinatorial numbers can also be dimensions of representations of the 0Hecke algebra, which cannot be lifted to the generic Hecke algebra or to the symmetric group. This is the case, for example, for linear extensions of a poset [8], which can be directly interpreted as free quasi-symmetric functions.

The aim of this paper is to apply this philosophy to the Bell polynomials. Their relation with symmetric functions is well-known, and easily extended to noncommutative symmetric functions. At this level, it is already possible to define a non-trivial $q$-analogue, which points toward the most promising direction for the next step. There is a natural choice of a representation of set partitions by permutations in FQSym which is compatible with this $q$-analogue. The Bell polynomials are then lifted to

Manuscript received 11th December 2017, revised 26th February 2018 and 12th June 2018, accepted 17 th June 2018.

KEYWORDs. Noncommutative symmetric functions, Quasi-symmetric functions, Bell polynomials, Dendriform algebras. 
polynomials in noncommuting variables $Y_{k}$, with coefficients in FQSym. One can then consider the quasi-symmetric functions $C_{I}$ which are the commutative images of the coefficients of the monomials $Y^{I}$. It turns out that they coincide with the dual immaculate basis of [2], up to mirror image of compositions.

The formal sum of these "free Bell polynomials" satisfies a simple functional equation in terms of the dendriform structure of FQSym. This allows us to obtain expressions of the dual immaculate basis similar to (but different from) those of Grinberg [14]. Actually, Grinberg works directly at the level of quasi-symmetric functions, and his formula comes in fact from the tridendriform structure of WQSym.

Finally, we discuss briefly the connection with the self-dual Hopf algebra of set partitions introduced by Rey in [35].

\section{Bell POLYNOMiAls AND NONCOMMUTATIVE SYMMETRIC FUNCTIONS}

2.1. Bell polynomials and symmetric functions. The classical Bell (exponential) polynomials are defined by

$$
B_{0}=1, B_{n+1}\left(y_{1}, \ldots, y_{n+1}\right)=\sum_{k=0}^{n}\left(\begin{array}{l}
n \\
k
\end{array}\right) B_{n-k} y_{k+1}
$$

or equivalently by the exponential generating series

$$
\mathcal{B}(t):=\sum_{n \geqslant 0} B_{n} \frac{t^{n}}{n !}=\exp \left(\sum_{k \geqslant 1} y_{k} \frac{t^{k}}{k !}\right) .
$$

This is reminescent of the relation between power-sums and complete symmetric functions [24]. The power sums are

$$
p_{n}=\sum_{i \geqslant 1} x_{i}^{n}
$$

and the complete symmetric function $h_{n}$ is the sum of all monomials of degree $n$, with generating series

$$
H(t)=\sum_{n \geqslant 0} h_{n} t^{n}=\prod_{i \geqslant 1} \frac{1}{1-t x_{i}}=\exp \left(\sum_{k \geqslant 1} p_{k} \frac{t^{k}}{k}\right)
$$

so that if one sets $y_{n}=(n-1) ! p_{n}$, then $B_{n}=n ! h_{n}$, and for $y_{n}=(-1)^{n-1}(n-1) ! p_{n}$, $B_{n}$ becomes $n ! e_{n}$, where the $e_{n}$ are the elementary symmetric functions.

2.2. Noncommutative Bell polynomials. This is easily extended to noncommutative symmetric functions. Recall [12] that the algebra Sym of noncommutative symmetric functions is freely generated ${ }^{(1)}$ by noncommuting indeterminates $S_{n}$ playing the role of the $h_{n}$. The noncommutative analogues of the $p_{n}$ are not canonically determined, and one possibility is to define noncommutative power-sums of the first kind $\Psi_{n}$ by orienting the Newton recursion as [12, Prop. 3.3]

$$
(n+1) S_{n+1}=\sum_{k=0}^{n} S_{n-k} \Psi_{k+1} .
$$

These power-sums correspond to the Dynkin elements in the descent algebras [12].

${ }^{(1)}$ All our algebras are taken over some unspecified field $\mathbb{K}$ of characteristic 0 , assumed to contain all indeterminates and formal power series implied by the context. 
Let $\left(Y_{n}\right)_{n \geqslant 1}$ be a sequence of noncommuting indeterminates. For a composition $I=\left(i_{1}, \ldots, i_{r}\right)$, let $Y^{I}=Y_{i_{1}} \cdots Y_{i_{r}}$. The recurrence (1) can be similarly oriented as

$$
B_{n+1}^{\prime}=\sum_{k=0}^{n}\left(\begin{array}{l}
n \\
k
\end{array}\right) B_{n-k}^{\prime} Y_{k+1} \text { or } B_{n+1}^{\prime \prime}=\sum_{k=0}^{n}\left(\begin{array}{l}
n \\
k
\end{array}\right) Y_{k+1} B_{n-k}^{\prime \prime} .
$$

Then, $B_{n}^{\prime}=n ! S_{n}$ for $Y_{n}=(n-1) ! \Psi_{n}$ and $B_{n}^{\prime \prime}=n ! S_{n}$ for $Y_{n}=(n-1) ! \bar{\Psi}_{n}$ (where the $\bar{\Psi}_{n}$ are defined by the opposite Newton recursion

$$
(n+1) S_{n+1}=\sum_{k=0}^{n} \bar{\Psi}_{k+1} S_{n-k},
$$

and correspond to the right-sided Dynkin elements). Such noncommutative Bell polynomials have been discussed in [36] (see also [31, 9]).

It is enough to describe one version, since the antiautomorphism $Y_{i} \mapsto Y_{i}$ exchanges $B_{n}^{\prime}$ and $B_{n}^{\prime \prime}$. Let us choose the second one. For now on, we set $B=B^{\prime \prime}$.

The first values are

$$
\begin{aligned}
B_{1}= & Y^{1} \\
B_{2}= & Y^{2}+Y^{11} \\
B_{3}= & Y^{3}+2 Y^{21}+Y^{12}+Y^{111} \\
B_{4}= & Y^{4}+3 Y^{31}+3 Y^{22}+3 Y^{211}+Y^{13}+2 Y^{121}+Y^{112}+Y^{1111} \\
B_{5}= & Y^{5}+4 Y^{41}+6 Y^{32}+6 Y^{311}+4 Y^{23}+8 Y^{221}+4 Y^{212}+4 Y^{2111} \\
& +Y^{14}+3 Y^{131}+3 Y^{122}+3 Y^{1211}+Y^{113}+2 Y^{1121}+Y^{1112}+Y^{11111}
\end{aligned}
$$

The coefficients have a simple combinatorial interpretation in terms of set partitions. We shall represent a set partition either by a set of sets or by any sequence of its parts separated by a vertical bar. For example, $\pi=\{\{3,4,7\},\{2,8\},\{1\},\{5,6\}\}$ will also be represented as $347|28| 1 \mid 56$.

For a set partition $\pi$ of $[n]$, let $\pi^{\sharp}$ be the set composition obtained by ordering the blocks of $\pi$ w.r.t. their minima, and let $K(\pi)$ be the composition recording the sizes of the blocks of $\pi^{\sharp}$. For example, if $\pi=347|28| 1 \mid 56$, then $\pi^{\sharp}=1|28| 347 \mid 56$, and $K(\pi)=(1,2,3,2)$. Then, the coefficient of $Y^{I}$ in $B_{n}$ is the number of set partitions $\pi$ of $[n]$ such that $K(\pi)=I$ (this follows by induction on $n$, as the first block of $\pi^{\sharp}$ is always the one containing 1). For example, the coefficient of $Y^{23}$ in $B_{5}$ enumerates the set of partitions

$$
12|345,13| 245,14|235,15| 234,
$$

whereas the coefficient of $Y^{32}$ in $B_{5}$ enumerates

$$
123|45,124| 35,125|34,134| 25,135|24,145| 23 .
$$

2.3. $q$-ANALOGUES. In $[20, \S 5.1]$, a noncommutative analogue of the classical transformation $p_{n} \mapsto\left(1-q^{n}\right) p_{n}$ of ordinary symmetric functions has been defined as the algebra automorphism

$$
\alpha_{q}: S_{n}(A) \mapsto S_{n}((1-q) A)=(1-q) \sum_{k=0}^{n-1}(-q)^{k} R_{1^{k}, n-k}
$$

(where the $R_{I}$ are the noncommutative ribbon Schur functions), which is the inverse of $([20, \S 6.1])$

$$
\beta_{q}: S_{n}(A) \mapsto S_{n}\left(\frac{A}{1-q}\right)
$$


where $\frac{A}{1-q}$ denotes the (genuine) alphabet $\left\{q^{i} a_{j}\right\}$ endowed with the order $q^{i} a_{j}<q^{k} a_{l}$ iff $i>k$ or $i=k$ and $j<l$.

In terms of the generating series of complete and elementary functions

(13) $\sigma_{t}(A):=\sum_{n \geqslant 0} t^{n} S_{n}(A)=\prod_{i \geqslant 1}\left(1-t a_{i}\right)^{-1}, \quad \lambda_{-t}(A)=\sum_{n \geqslant 0}(-t)^{n} \Lambda_{n}(A)=\sigma_{t}(A)^{-1}$

these transformations read

$$
\sigma_{t}\left(\frac{A}{1-q}\right)=\prod_{k \geqslant 0}^{\leftarrow} \sigma_{q^{k} t}(A)=\cdots \sigma_{q^{2} t}(A) \sigma_{q t}(A) \sigma_{t}(A)
$$

and

$$
\sigma_{t}((1-q) A)=\lambda_{-q t}(A) \sigma_{t}(A)
$$

It follows from (11) that the Dynkin power-sums $\Psi_{n}$ are limiting cases of the transformed complete functions (see [20] for an explanation of the notation)

$$
\Psi_{n}=\lim _{q \rightarrow 1} \frac{S_{n}((1-q) A)}{1-q}
$$

and similarly

$$
(-1)^{n-1} \Psi_{n}=\lim _{q \rightarrow 1} \frac{\Lambda_{n}((1-q) A)}{1-q} .
$$

Indeed, it is known that $\Psi_{n}=\sum_{k=0}^{n-1}(-1)^{k} R_{1^{k}, n-k}$, and

$$
\begin{aligned}
\sum_{n \geqslant 0}(-t)^{n} \Lambda_{n}((1-q) A) & =\sigma_{t}((1-q) A)^{-1}=\left(\lambda_{-q t}(A) \sigma_{t}(A)\right)^{-1} \\
& =\lambda_{-t}(A) \sigma_{q t}(A)=\sigma_{1}((q-1) t A)=\sigma_{q t}\left(\left(1-\frac{1}{q}\right) A\right)
\end{aligned}
$$

so that

$$
\Lambda_{n}((1-q) A)=(-1)^{n}(q-1) \sum_{k=0}^{n-1}\left(-\frac{1}{q}\right)^{n-1-k} R_{1^{k}, n-k} .
$$

Replacing $A$ by $A /(1-q)$, one can define $q$-analogues of $B_{n}^{\prime}$ and $B_{n}^{\prime \prime}$ by

$$
B_{n}^{\prime}(q)=(q)_{n} S_{n}\left(\frac{A}{1-q}\right) \text { for } Y_{n}=(q)_{n-1} S_{n}(A)
$$

and

$$
B_{n}^{\prime \prime}(q)=(q)_{n} \Lambda_{n}\left(\frac{A}{1-q}\right) \text { for } Y_{n}=(q)_{n-1} \Lambda_{n}(A),
$$

where $(q)_{n}:=\prod_{i=1}^{n}\left(1-q^{i}\right)$.

This amounts to defining $B_{n}^{\prime}(q)$ by the recursion

$$
B_{n}^{\prime}(q)=\sum_{k=0}^{n-1} q^{k}\left[\begin{array}{c}
n-1 \\
k
\end{array}\right]_{q} B_{k}^{\prime}(q) Y_{n-k} \quad\left(\text { and } B_{0}=1\right) .
$$


Indeed,

$$
\begin{aligned}
B_{n}^{\prime}(q) & =(q)_{n} S_{n}\left(\left(\cdots+q^{2} A+q A\right)+A\right) \\
& =(q)_{n} S_{n}\left(\frac{q A}{1-q}+A\right) \\
& =(q)_{n} \sum_{k=0}^{n} S_{k}\left(\frac{q A}{1-q}\right) S_{n-k}(A) \\
& =(q)_{n} \sum_{k=0}^{n} q^{k} S_{k}\left(\frac{A}{1-q}\right) S_{n-k}(A) \\
& =\sum_{k=0}^{n-1} \frac{(q)_{n}}{(q)_{k}} q^{k} B_{k}^{\prime}(q) \frac{Y_{n-k}}{(q)_{n-k-1}}+(q)_{n} q^{n} S_{n}\left(\frac{A}{1-q}\right) \\
& =\left(1-q^{n}\right) \sum_{k=0}^{n-1}\left[\begin{array}{c}
n-1 \\
k
\end{array}\right]_{q} q^{k} B_{k}^{\prime}(q) Y_{n-k}+q^{n} B_{n}^{\prime}(q) .
\end{aligned}
$$

The same argument yields for $B_{n}^{\prime \prime}(q)$

$$
B_{n}^{\prime \prime}(q)=\sum_{k=0}^{n-1} q^{k} Y_{n-k}\left[\begin{array}{c}
n-1 \\
k
\end{array}\right]_{q} B_{k}^{\prime \prime}(q) \quad\left(\text { and } B_{0}=1\right) .
$$

The first values are

$$
\begin{aligned}
B_{1}^{\prime}= & Y^{1} \\
B_{2}^{\prime}= & Y^{2}+q Y^{11} \\
B_{3}^{\prime}= & Y^{3}+q^{2} Y^{21}+\left(q^{2}+q\right) Y^{12}+q^{3} Y^{111} \\
B_{4}^{\prime}= & Y^{4}+q^{3} Y^{31}+\left(q^{4}+q^{3}+q^{2}\right) Y^{22}+q^{5} Y^{211} \\
& +\left(q^{3}+q^{2}+q\right) Y^{13}+\left(q^{5}+q^{4}\right) Y^{121}+\left(q^{5}+q^{4}+q^{3}\right) Y^{112}+q^{6} Y^{1111}
\end{aligned}
$$

and

$$
\begin{aligned}
B_{1}^{\prime \prime}= & Y^{1} \\
B_{2}^{\prime \prime}= & Y^{2}+q Y^{11} \\
B_{3}^{\prime \prime}= & Y^{3}+\left(q^{2}+q\right) Y^{21}+q^{2} Y^{12}+q^{3} Y^{111} \\
B_{4}^{\prime \prime}= & Y^{4}+\left(q^{3}+q^{2}+q\right) Y^{31}+\left(q^{4}+q^{3}+q^{2}\right) Y^{22}+\left(q^{5}+q^{4}+q^{3}\right) Y^{211} \\
& +q^{3} Y^{13}+\left(q^{5}+q^{4}\right) Y^{121}+q^{5} Y^{112}+q^{6} Y^{1111} .
\end{aligned}
$$

Setting $Y_{i}=1$ in $B_{n}^{\prime}$ or in $B_{n}^{\prime \prime}$, one obtains the triangle A188919 of [37]:

$$
\begin{aligned}
& b_{1}(q)=1 \\
& b_{2}(q)=1+q \\
& b_{3}(q)=1+q+2 q^{2}+q^{3} \\
& b_{4}(q)=1+q+2 q^{2}+4 q^{3}+3 q^{4}+3 q^{5}+q^{6} \\
& b_{5}(q)=1+q+2 q^{2}+4 q^{3}+7 q^{4}+8 q^{5}+9 q^{6}+9 q^{7}+6 q^{8}+4 q^{9}+q^{10} .
\end{aligned}
$$

The On-Line Encyclopedia of Integer Sequences [37] suggests that the coefficient of $q^{k}$ in $b_{n}(q)$ should be the number of permutations of length $n$ with $k$ inversions that avoid the dashed pattern $1-32$ [1]. The proof of this fact will follow from a refined 
interpretation for the coefficient of $Y^{I}$ in $B_{n}^{\prime}(q)$ (Proposition 3.4 below). Indeed, it follows from (23) that this coefficient is ${ }^{(2)}$

$$
\prod_{k=2}^{\ell(I)}\left[\begin{array}{c}
i_{1}+\cdots+i_{k}-1 \\
i_{k}-1
\end{array}\right]_{q} q^{i_{1}+\cdots+i_{k-1}} .
$$

This suggests that the counting of $1-32$-avoiding permutations can be refined according to their descent sets. To do this, we have to replace the binomial coefficients in the recurrence by formal sums of permutations in an appropriate algebra.

As we have seen, the Bell polynomials are essentially the expressions of the complete homogeneous symmetric functions on suitably normalized power sums. This expression is classically given by a determinant, which has been lifted to the noncommutative case in [9, Thm. 2.5] as a quasideterminant. Expressions of the $S_{n}$ as quasideterminants in the $\Psi_{n}$ have been given in [12], and have been extended to the $q$-analogues $\Theta_{n}(q)=(1-q)^{-1} S_{n}((1-q) A)$ of the $\Psi_{n}$ in [20, Prop. 5.5]. A straightforward manipulation of this quasideterminant yields the following $q$-analogue of $[9$, Thm. 2.5]:

Proposition 2.1. $B_{n}^{\prime}(q)$ is given by the quasideterminant

$$
B_{n}^{\prime}(q)=\left|\mathbb{B}_{n}^{\prime}\right|_{1 n}
$$

where $\mathbb{B}_{n}^{\prime}$ is the $n \times n$ matrix whose subdiagonal elements are -1 , the lower elements are all zero, and the elements on and above the diagonal are

$$
\left(\mathbb{B}_{n}^{\prime}\right)_{i j}=q^{i-1}\left[\begin{array}{c}
n-(n-j+1) \\
j-i
\end{array}\right]_{q} Y_{j-i+1}(\text { for } i \leqslant j) .
$$

Proof. Recall that the $q$-factorial is defined by $[n]_{q} !=[n]_{q}[n-1]_{q} \cdots[1]_{q}$, where $[n]_{q}=\left(1-q^{n}\right) /(1-q)$. Applying the inverse transformation $\beta_{q}$, it is clear that $B_{n}^{\prime}(q)$ is also the polynomial expressing $[n]_{q} ! S_{n}(A)$ in terms of the $Y_{k}=[k-1]_{q} ! \Theta_{k}(q)$ (which are obviously a set of free generators of Sym on $\mathbb{K}(q))$. Indeed, setting

$$
B_{n}^{\prime}(q)=\sum_{I \models n} c_{I}(q) Y^{I}=\sum_{I \models n} c_{I}(q) \prod_{k=1}^{\ell(I)}(q)_{i_{k}-1} S_{i_{k}}(A)
$$

we have

$$
\begin{aligned}
(q)_{n} S_{n}(A) & =\sum_{I \models n} c_{I}(q) \prod_{k=1}^{\ell(I)}(q)_{i_{k}-1} S_{i_{k}}((1-q) A) \\
& =\sum_{I \models n} c_{I}(q) \prod_{k=1}^{\ell(I)}(q)_{i_{k}-1}(1-q)^{\ell(I)} \cdot \Theta^{I}(q)
\end{aligned}
$$

and dividing by $(1-q)^{n}$ yields

$$
\begin{aligned}
{[n]_{q} ! S_{n}(A) } & =\sum_{I \models n} c_{I}(q) \prod_{k=1}^{\ell(I)}\left[i_{k}-1\right]_{q} !(1-q)^{i_{k}-1} \cdot(1-q)^{\ell(I)} \Theta^{I}(q) \\
& =\sum_{I \models n} c_{I}(q) \prod_{k=1}^{\ell(I)}\left[i_{k}-1\right]_{q} ! \cdot \Theta^{I}(q) .
\end{aligned}
$$

${ }^{(2)}$ This is a $q$-analogue of the r.h.s. of $[31,(78)]$. 
In the quasideterminant (78) of [20], expressing $S_{n}(A)$ in terms of $\Theta_{n}(q)$

$$
[n]_{q} S_{n}(A)=\left|\begin{array}{ccccc}
\Theta_{1}(q) & \Theta_{2}(q) & \ldots & \Theta_{n-1}(q) & \overline{\Theta_{n}(q)} \\
-[1]_{q} & q \Theta_{1}(q) & \ldots & q \Theta_{n-2}(q) & q \Theta_{n-1}(q) \\
0 & -[2]_{q} & \ldots & q^{2} \Theta_{n-3}(q) & q^{2} \Theta_{n-2}(q) \\
\vdots & \vdots & \vdots & \vdots & \vdots \\
0 & 0 & \ldots & -[n-1]_{q} & q^{n-1} \Theta_{1}(q)
\end{array}\right| .
$$

multiply column $j$ by $[j-1]_{q}$ ! and divide row $i$ by $[i-1]_{q}$ !. These operations do not change the value of the quasideterminant, except for the last column, which yields a factor $[n-1]_{q}$ !, so that the 1.h.s. of $[20,(78)]$ is now $[n]_{q} ! S_{n}$. Replacing each entry $\Theta_{k}(q)$ by $\frac{1}{[k-1]_{q} !} Y_{k}$ yields the desired expression.

For example,

$$
B_{4}^{\prime}(q)=\left|\begin{array}{ccccc}
Y_{1} & Y_{2} & Y_{3} & {\left[Y_{4}\right.} \\
-1 & q\left[\begin{array}{l}
2 \\
0
\end{array}\right]_{q} Y_{1} & q\left[\begin{array}{l}
2 \\
1
\end{array}\right]_{q} Y_{2} & q\left[\begin{array}{l}
2 \\
2
\end{array}\right]_{q} Y_{3} \\
0 & -1 & q^{2}\left[\begin{array}{l}
3 \\
0
\end{array}\right]_{q} Y_{1} & q^{2}\left[\begin{array}{l}
3 \\
1
\end{array}\right]_{q} Y_{2} \\
0 & 0 & -1 & q^{3}\left[\begin{array}{l}
4 \\
0
\end{array}\right]_{q} Y_{1}
\end{array}\right| .
$$

Note that Equation (77) of [20] allows one to express $Y_{n}$ in terms of the $B_{k}$, as in $[9, \S 3.2 .6]$.

\section{Quasi-Symmetric analogues of the COEFFicients}

3.1. FreE QUASI-SYMMETRIC FUNCTIONS AND THEIR DENDRIFORM STRUCTURE. Recall that for a totally ordered alphabet $A, \operatorname{FQSym}(A)$ is the algebra spanned by the noncommutative power series [8]

$$
\mathbf{G}_{\sigma}(A):=\sum_{\substack{w \in A^{n} \\ \operatorname{std}(w)=\sigma}} w
$$

where $\sigma$ is a permutation in the symmetric group $\mathfrak{S}_{n}$ and $\operatorname{Std}(w)$ denotes the standardization of the word $w$, i.e., the unique permutation having the same inversions as $w$.

All countably infinite alphabets lead to isomorphic algebras. In the sequel, we fix one such alphabet $A$ and denote the resulting algebra $\mathbf{F Q S y m}(A)$ simply by FQSym.

The multiplication rule is the same as in the Malvenuto-Reutenauer algebra [25], to which FQSym is therefore isomorphic ${ }^{(3)}$

$$
\mathbf{G}_{\alpha} \mathbf{G}_{\beta}=\sum_{\gamma \in \alpha * \beta} \mathbf{G}_{\gamma},
$$

where the convolution $\alpha * \beta$ of $\alpha \in \mathfrak{S}_{k}$ and $\beta \in \mathfrak{S}_{l}$ is defined as the sum in the group algebra of $\mathfrak{S}_{k+l}$ in [25]

$$
\alpha * \beta=\sum_{\substack{\gamma=u v \\ \operatorname{Std}(u)=\alpha ; \operatorname{Std}(v)=\beta}} \gamma .
$$

Permutations are here interpreted as words, and $u v$ means concatenation. The result is interpreted here as a set. Indeed, there are no multiplicities in this sum, and the

\footnotetext{
${ }^{(3)}$ The interest of this isomorphism resides in the fact that the Hopf algebra structure is then induced by the ordered sum of alphabets, as for noncommutative symmetric functions.
} 
number of terms in $\mathbf{G}_{\alpha} \mathbf{G}_{\beta}$ is $\left(\begin{array}{c}k+l \\ k\end{array}\right)$. To reduce this number to $\left(\begin{array}{c}k+l-1 \\ k\end{array}\right)$ and relate to the Bell induction concerning $B_{n}^{\prime \prime}$, we can split the product according to the dendriform structure of FQSym.

The dendriform structure of FQSym, originally defined in [23], can be seen as inherited from the tridendriform structure of the algebra of noncommutative formal power series over $A$ defined in $[26,27]$. Define bilinear operators on nonempty words $u, v$ by

$$
\begin{aligned}
& u \prec v= \begin{cases}u v & \text { if } \max (v)<\max (u) \\
0 & \text { otherwise, }\end{cases} \\
& u \succ v= \begin{cases}u v & \text { if } \max (v) \geqslant \max (u) \\
0 & \text { otherwise. }\end{cases}
\end{aligned}
$$

Note that the operator $\succ$ here is $\succ$ +o of [26, 27]. Also, our conventions differ from those of Grinberg [14, Def. 6.1] who uses min instead of max, and $>$ and $<$ instead of $<$ and $\geqslant$. This yields

$$
\mathbf{G}_{\alpha} \mathbf{G}_{\beta}=\mathbf{G}_{\alpha} \prec \mathbf{G}_{\beta}+\mathbf{G}_{\alpha} \succ \mathbf{G}_{\beta},
$$

where

$$
\begin{aligned}
& \mathbf{G}_{\alpha} \prec \mathbf{G}_{\beta}=\sum_{\substack{\gamma=u v \in \alpha * \beta \\
|u|=|\alpha| ; \max (v)<\max (u)}} \mathbf{G}_{\gamma}, \\
& \mathbf{G}_{\alpha} \succ \mathbf{G}_{\beta}=\sum_{\substack{\gamma=u v \in \alpha * \beta \\
|u|=|\alpha| ; \max (v) \geqslant \max (u)}} \mathbf{G}_{\gamma} .
\end{aligned}
$$

Then $x=\mathbf{G}_{1}$ generates a free dendriform algebra in FQSym, isomorphic to PBT, the Loday-Ronco algebra of planar binary trees [22, Prop. 3.7] and [23, Prop. 5.3]. The notations used here are those of [16], where more details can be found.

Recall that the shuffle product $\omega$ is recursively defined by

$$
u a \varpi v b=(u a ш v) b+(u ш v b) a,
$$

where $a, b$ are letters and $u, v$ words. The scalar 1 (representing the empty word) is the neutral element. The shuffle can be split into two half-products $\prec$ and $\succ$ defined by

$$
u a \prec v b=(u ш v b) a, u a \succ v b=(u a ш v) b,
$$

We shall not need dendriform products involving 1 and thus leave these undefined.

In terms of the dual basis $\mathbf{F}_{\sigma}:=\mathbf{G}_{\sigma^{-1}}$,

$$
\begin{aligned}
\mathbf{F}_{\alpha} \mathbf{F}_{\beta} & =\sum_{\gamma \in \alpha \amalg \beta[k]} \mathbf{F}_{\gamma}, \\
\mathbf{F}_{\alpha} \prec \mathbf{F}_{\beta} & =\sum_{\gamma \in \alpha \prec \beta[k]} \mathbf{F}_{\gamma}, \\
\mathbf{F}_{\alpha} \succ \mathbf{F}_{\beta} & =\sum_{\gamma \in \alpha \succ \beta[k]} \mathbf{F}_{\gamma},
\end{aligned}
$$

where $\alpha \in \mathfrak{S}_{k}, \beta[k]$ denotes $\beta$ with its entries shifted by $k$.

There is an inclusion of Hopf algebras $\mathbf{S y m} \hookrightarrow \mathbf{P B T}$ of noncommutative symmetric functions into PBT [22, 23], which is given by

$$
S_{n}=(\ldots((x \succ x) \succ x) \ldots) \succ x \quad(n \text { times }) .
$$

Indeed, the r.h.s is the sum of nondecreasing words, i.e., $\mathbf{G}_{1 \ldots n}=S_{n}$. 
3.2. A DENDRIForm Lift of THE Bell RECURRENCE. We shall define the free Bell polynomials as elements of $\mathbb{K}\left\langle Y_{1}, Y_{2}, \ldots\right\rangle \otimes$ FQSym by

$$
\boldsymbol{B}_{0}=1, \quad \boldsymbol{B}_{n+1}=\sum_{k=0}^{n} Y_{k+1} S_{k+1} \prec \boldsymbol{B}_{n-k},
$$

the $\otimes$ sign being omitted for notational convenience, and $\prec$ is actually $\cdot \otimes \prec$. Recall also that $S_{k+1}=\mathbf{G}_{1 \ldots k+1}$. The first $\boldsymbol{B}_{n}$ are

$$
\begin{aligned}
\boldsymbol{B}_{0}= & 1 \\
\boldsymbol{B}_{1}= & Y^{1} \mathbf{G}_{1} \\
\boldsymbol{B}_{2}= & Y^{2} \mathbf{G}_{12}+Y^{11} \mathbf{G}_{21} \\
\boldsymbol{B}_{3}= & Y^{3} \mathbf{G}_{123}+Y^{21}\left(\mathbf{G}_{132}+\mathbf{G}_{231}\right)+Y^{12} \mathbf{G}_{312}+Y^{111} \mathbf{G}_{321} \\
\boldsymbol{B}_{4}= & Y^{4} \mathbf{G}_{1234}+Y^{31}\left(\mathbf{G}_{1243}+\mathbf{G}_{1342}+\mathbf{G}_{2341}\right) \\
& +Y^{22}\left(\mathbf{G}_{1423}+\mathbf{G}_{2413}+\mathbf{G}_{3412}\right)+Y^{211}\left(\mathbf{G}_{1432}+\mathbf{G}_{2431}+\mathbf{G}_{3421}\right) \\
& +Y^{13} \mathbf{G}_{4123}+Y^{121}\left(\mathbf{G}_{4132}+\mathbf{G}_{4231}\right)+Y^{112} \mathbf{G}_{4312}+Y^{1111} \mathbf{G}_{4321} .
\end{aligned}
$$

Clearly, for each $n \geqslant 0, \boldsymbol{B}_{n}$ is a (left) FQSym-linear combination of the $Y^{I}$ for various compositions $I$ of $n$. The coefficient $C_{I}(A)$ of $Y^{I}$ in $\boldsymbol{B}_{n}$ is by definition in FQSym but is in fact in PBT:

LEMMA 3.1. Let $I=\left(i_{1}, \ldots, i_{r}\right)$ be a composition. Then $C_{I}(A)$ is equal to $\mathbf{P}_{T}$ where $T$ is the right-comb tree whose left branches have sizes from top to bottom equal to $i_{1}$, $i_{2}, \ldots, i_{r}$.

Proof. The coefficient $C_{I}(A)$ is by definition equal to

$$
S_{i_{1}} \prec\left(S_{i_{2}} \prec\left(\cdots\left(S_{i_{r-1}} \prec S_{i_{r}}\right) \cdots\right) .\right.
$$

Let $n$ be $i_{1}+\cdots+i_{r}$ and expand that product in FQSym on the $\mathbf{G}$ basis. Consider any permutation $\tau$ such that $\mathbf{G}_{\tau}$ occurs in the expansion. By definition of $\prec$, the first $i_{1}$ letters of $\tau$ are increasing and the $i_{1}$-th letter is $n$, then followed by a word whose standardization $\sigma$ belongs to $\left.S_{i_{2}} \prec\left(\cdots\left(S_{i_{r-1}} \prec S_{i_{r}}\right)\right) \cdots\right)$. Hence, by induction on $r$, the decreasing tree (see $[22,16])$ of all these elements is obtained by grafting the shape of the decreasing tree of $\sigma$ to the right of the root of a left branch of size $i_{1}$. Conversely, since any permutation of decreasing tree $T$ belongs to the left product (60), this product is equal to $\mathbf{P}_{T}$.

EXAMPLE 3.2. The coefficient of $Y^{221}$ in $\boldsymbol{B}_{5}$ is

$$
\begin{aligned}
\mathbf{G}_{12} \prec\left(\mathbf{G}_{132}+\mathbf{G}_{231}\right)= & \mathbf{G}_{15243}+\mathbf{G}_{25143}+\mathbf{G}_{35142}+\mathbf{G}_{45132} \\
& +\mathbf{G}_{15342}+\mathbf{G}_{25341}+\mathbf{G}_{35241}+\mathbf{G}_{45231} \\
= & \mathbf{P}
\end{aligned}
$$

For a set partition $\pi$ of $[n]$, let $\pi^{\text {b }}$ be the set composition obtained by ordering the blocks w.r.t. their maximal values in decreasing order, and let $K(\pi)$ be the composition recording the lengths of these blocks. Let also $\hat{\pi}^{b}$ be the permutation obtained by reading the blocks in this order, where each block is read from the smallest to the largest element.

EXAMPLE 3.3. If $\pi=347|28| 1 \mid 56$, we have $\pi^{b}=28|347| 56 \mid 1$, so that $\hat{\pi}^{b}=28347561$, and $K(\pi)=(2,3,2,1)$. 
Proposition 3.4. The coefficient of $Y^{I}$ in $\boldsymbol{B}_{n}$ is the sum of all $\mathbf{G}_{\hat{\pi}^{b}}$ where $\pi$ ranges over set partitions such that $K(\pi)=I$ :

$$
\boldsymbol{B}_{n}=\sum_{\pi \vdash[n]} Y^{K(\pi)} \mathbf{G}_{\hat{\pi}^{b}} .
$$

Proof. This follows from the product rule (50). Indeed, as we have already seen, this coefficient is $S_{i_{1}} \prec\left(S_{i_{2}} \prec\left(\cdots\left(S_{i_{r-1}} \prec S_{i_{r}}\right)\right) \cdots\right)$, which is the sum of $\mathbf{G}_{\sigma}$ over all permutations of the form $\sigma=u_{1} u_{2} \cdots u_{r}$, where each $u_{j}$ is an increasing word of length $i_{j}$ whose last letter is greater than all letters to its right. But these permutations are precisely the $\hat{\pi}^{b}$ for the set partitions $\pi$ such that $K(\pi)=I$.

These permutations $\hat{\pi}^{b}$ are not those avoiding the pattern $1-32$ as above, but those avoiding the pattern $21-3$. As observed in [6, Prop. 1], there is a statisticpreserving bijection between both classes (in this case, the Schützenberger involution, which preserves the inversion number). Since the inverse major index and the inversion number are equidistributed on the set of permutations having a decreasing tree of a given shape [18, Thm. 2.7], the generating polynomial of inv on $1-32$ or of imaj on $21-3$-avoiding permutations with descent composition $I$ is

$$
c_{I}(q)=C_{I}\left(\frac{1}{1-q}\right)
$$

where the alphabet $\frac{1}{1-q}$ (the principal specialization of FQSym) is defined by

$$
A \mapsto\left\{q^{n} \mid n \geqslant 0\right\} \text { ordered by } q^{i}<q^{j} \text { iff } i>j .
$$

Indeed,

$$
C_{I}\left(\frac{1}{1-q}\right)=\sum_{K(\pi)=I} \mathbf{G}_{\hat{\pi}^{b}}\left(\frac{1}{1-q}\right)
$$

and

$$
\mathbf{G}_{\sigma}\left(\frac{1}{1-q}\right)=F_{I}\left(\frac{1}{1-q}\right)
$$

where $I$ is the descent composition of $\sigma^{-1}$. It is well-known (see [13, Lem. 5.2] or [12, Prop. 5.10]) the specializations of the fundamental quasi-symmetric functions are

$$
F_{I}\left(\frac{1}{1-q}\right)=\frac{q^{\operatorname{maj}(I)}}{(q)_{n}} .
$$

where $\operatorname{maj}(I)=\operatorname{maj}(\tau)$ for any permutation $\tau$ of shape $I$. Thus, specializing the FQSym coefficients in $\boldsymbol{B}_{n}$, we obtain

$$
(q)_{n} \boldsymbol{B}_{n}\left(\frac{1}{1-q}\right)=B_{n}^{\prime \prime}(q)
$$

since by definition, the recurrence (58) is a lift of (30).

Now that we understand that $c_{I}(q)$ is the principal specialization of a quasisymmetric function, we can replace it by the commutative image $C_{I}(X)$ of $C_{I}(A)$ in QSym. Recall that if the letters $a_{i}$ of our underlying alphabet $A$ are replaced by commuting variables $x_{i}$, then $\mathbf{F}_{\sigma}$ becomes the fundamental quasi-symmetric function $F_{I}(X)$ where $I$ is the descent composition of $\sigma$.

EXAMPLE 3.5. Recording the recoil compositions of the permutations occuring in $C_{221}$, we find

$$
C_{221}(X)=F_{1121}+F_{1211}+F_{122}+F_{131}+F_{212}+2 F_{221}+F_{311}
$$


This may be compared with the dual immaculate basis of [2]:

$$
\mathfrak{S}_{221}^{*}=F_{1121}+F_{113}+F_{1211}+2 F_{122}+F_{131}+F_{212}+F_{221} .
$$

THEOREM 3.6. Define the bar involution on QSym by $\overline{F_{I}}=F_{\bar{I}}$, where for $I=$ $\left(i_{1}, \ldots, i_{r}\right), \bar{I}=\left(i_{r}, \ldots, i_{1}\right)$ denotes the mirror composition. Then, the dual immaculate basis is given by

$$
\mathfrak{S}_{I}^{*}=\overline{C_{I}(X)}
$$

Proof. According to [2, Prop. 3.37],

$$
\mathfrak{S}_{I}^{*}=\sum_{T} F_{D(T)}
$$

where the sum runs over all standard immaculate tableaux of shape $I$, and $D(T)$ denotes the descent composition of $T$ as defined in [2]. This should not be confused with the usual descent composition of a permutation $\sigma$, which will be denoted by $C(\sigma)$.

A standard immaculate tableau $T$ of shape $I$ is a planar representation of a set partition $\pi=\left(\pi_{1}, \ldots, \pi_{r}\right)$, whose blocks have been ordered in such a way that $\min \left(\pi_{1}\right)<\min \left(\pi_{2}\right)<\cdots<\min \left(\pi_{r}\right)$, and such that $\left|\pi_{j}\right|=i_{j}$.

For example, the standard immaculate tableaux of shape $I=(2,2,1)$ are

\begin{tabular}{|c|c|c|c|}
\hline 1 & 5 & 1 & 4 \\
\hline 2 & 3 & 2 & 5 \\
\hline 4 & & 3 & \\
\hline
\end{tabular}

\begin{tabular}{|c|c|c|c|}
\hline 1 & 4 & 1 & 3 \\
\hline 2 & 3 & 2 & 5 \\
\hline 5 & & 4 & \\
\hline
\end{tabular}

$$
\begin{array}{|l|l|}
\hline 1 & 5 \\
\hline 2 & 4 \\
\hline 3 & \multicolumn{1}{|l}{} \\
\cline { 1 - 1 }
\end{array}
$$

\begin{tabular}{|c|c|c|c|}
\hline 1 & 3 & 1 & 2 \\
\hline 2 & 4 & 3 & 5 \\
\hline 5 & & 4 & \\
\hline
\end{tabular}

\begin{tabular}{|l|l|}
\hline 1 & 2 \\
\hline 3 & 4 \\
\hline 5 & \multicolumn{1}{|c}{} \\
\cline { 1 - 1 } &
\end{tabular}

The descent composition $D(T)$ encodes the set $\{i \mid i+1$ is in a lower row $\}$, which is therefore the recoil set of the permutation $\hat{T}$ obtained by reading the rows of $T$ from bottom to top and from left to right.

The descent compostions $D(T)$ of the above tableaux are

$$
113,122,1121,131,122,1211,212,221 .
$$

These are the recoil compositions of the permutations $\hat{T}$

$$
32415,42315,32514,52314,42513,52413,43512,53412,
$$

whose descent compositions are obviously always $\bar{I}=(1,2,2)$.

The Schützenberger involution $\nu$ sends a permutation $\sigma \in \mathfrak{S}_{n}$ to the permutation $\sigma^{\prime}=\nu(\sigma)$ obtained by replacing each entry $i$ of $\sigma$ by $n+1-i$ and then reading the resulting word from right to left. In other words, $\sigma^{\prime}=\omega \sigma \omega$, where $\omega=n n-1 \cdots 21$.

The effect of $\nu$ on descent compositions is the mirror image $C\left(\sigma^{\prime}\right)=\overline{C(\sigma)}$. Thus, for any standard immmaculate tableau $T$ of shape $I$ and descent composition $J$, applying $\nu$ to the permutation $\sigma=\nu(\hat{T})$ yields

$$
C(\sigma)=C(\nu(\hat{T}))=\overline{C(\hat{T})}=\overline{\bar{I}}=I
$$

while

$$
C\left(\sigma^{-1}\right)=\overline{D(\hat{T})}=\bar{J} .
$$

Moreover, $\nu$ exchanges the maxima and the minima, so that the rows of the ribbon diagram of $\sigma$ form the blocks of a set partition $\pi=\left(\pi_{1}, \ldots, \pi_{r}\right)$ ordered in such a way that $\max \left(\pi_{1}\right)>\max \left(\pi_{2}\right)>\cdots>\max \left(\pi_{r}\right)$, and $\left|\pi_{j}\right|=i_{j}$. That is, $\sigma=\hat{\pi}^{b}$ for a set partition such that $K(\pi)=I$. Therefore,

$$
\mathfrak{S}_{I}^{*}=\sum_{K(\pi)=I} \overline{F_{K\left(\hat{\pi}^{b}\right)}}=\overline{C_{I}(X)} .
$$


On our running example, the permutations $\nu(\hat{T})$ are

$$
\text { 15243, 15342, 25143, 25341, 35142, 35241, 45132, } 45231 .
$$

Their recoil compositions are, in order

$$
\text { 311, 221, 1211, 131, 221, 1121, 212, } 122 .
$$

Note 3.7. Theorem 3.6 provides an expression of the dual immaculate basis very similar to the one obtained by Grinberg [14]. However, our dendriform operations are different. The relations between both constructions will be clarified in the forthcoming section.

Corollary 3.8. The $C_{I}(X)$ form a basis of QSym, so that the $\mathbf{P}_{T}$ indexed by right combs form a section of the projection $\mathbf{P B T} \rightarrow Q S y m$.

COROllary 3.9. $c_{I}(q)$ is given by the Björner-Wachs q-hook-length formula [4]: the inversion polynomial of the set of permutations having a decreasing tree of shape $T$ is given by the same hook length formula as for the inverse major index, which is

$$
\sum_{\mathcal{T}(\sigma)=T} q^{l(\sigma)}=[n]_{q} ! \prod_{v \in T} \frac{q^{\delta_{v}}}{\left[h_{v}\right]_{q}},
$$

where $v$ runs over the vertices of $T, h_{v}$ is the number of vertices of the subtree with root $v$, and $\delta_{v}$ is the number of vertices in the right subtree of $v$.

See [18] for a short proof of the version used here.

EXAMPLE 3.10. For $I=221$, the hook-lengths $h_{v}$ of the right comb are $5,3,1,1,1$, and the cardinalities of the right subtrees are 3,1 . Hence,

$$
c_{221}(q)=[5]_{q} ! \frac{q^{3+1}}{[5]_{q}[3]_{q}[1]_{q}^{3}}=q^{4}+2 q^{5}+2 q^{6}+2 q^{7}+q^{8} .
$$

Applying the Schützenberger involution $\nu$ to $C_{I}$ amounts to sending $\mathbf{G}_{\sigma}$ to $\check{\mathbf{G}}_{\sigma}=$ $\mathbf{G}_{\omega \sigma \omega}$, so that we can also reformulate Theorem 3.6 as

$$
\check{C}_{I}(X)=\mathfrak{S}_{I}^{*} \text {. }
$$

CoRollary 3.11 ([3]). $\overline{\mathfrak{S}_{I}^{*}}$ is the characteristic of an indecomposable 0-Hecke algebra module.

Proof. The inverses of the permutations occuring in $C_{I}(A)$ are the linear extensions of a poset (a binary tree in this case), hence form the basis of a 0-Hecke module, see Section 3.9 of [8]. Thus, the commutative image $C_{I}(X)=\overline{\mathfrak{S}_{I}^{*}}$ of $C_{I}(A)$ in QSym is the characteristic of this module. The same is true of their images by the Schützenberger involution, the poset being now a binary tree turned upside-down. Moreover, these modules are indecomposable, since they are of the form $\mathbf{N}_{\sigma}$ described in $[8$, Def. 4.3]. Indeed, the right-comb tree associated with a composition $I$ is the shape of the decreasing tree of the maximal permutation $\omega(I)$ of the descent class $I$. This permutation (which is sent to $\omega(\bar{I})$ by the Schützenberger involution) spans the (onedimensional) socle of the module, which must be contained in any submodule, so that no submodule can be a direct summand.

See [21] or [38] for an introduction to the representation theory of the 0-Hecke algebra. 


\section{The DUAL ImmaCUlate Basis}

4.1. Half-ShUfFles AND FQSym. The shuffle product on $\mathbb{K}\langle A\rangle$ can be recursively defined by (52):

$$
u a ш v b=(u a ш v) b+(u ш v b) a
$$

or symmetrically by

$$
a u ш b v=a(u ш b v)+b(a u ш v),
$$

where $a, b \in A$ and $u, v \in A^{*}$. The half-shuffles are also known as chronological products. Both ways of splitting the shuffle can be used to define a dendriform structure on FQSym. In this section, we shall use the second possibility (84) and set

$$
a u \prec^{\prime} b v=a(u ш b v), \text { and } a u \succ^{\prime} b v=b(a u ш v) .
$$

If $\gamma$ is a linear combination of some permutations $\rho$, we write for short $\mathbf{F}_{\gamma}$ for the same linear combination of the $\mathbf{F}_{\rho}$. On FQSym, we set, for $\sigma \in \mathfrak{S}_{k}$ and $\tau \in \mathfrak{S}_{l}$

$$
\mathbf{F}_{\sigma} \prec^{\prime} \mathbf{F}_{\tau}=\mathbf{F}_{\sigma \prec^{\prime} \tau[k]} \text {, and } \mathbf{F}_{\sigma} \succ^{\prime} \mathbf{F}_{\tau}=\mathbf{F}_{\sigma \succ^{\prime} \tau[k]} .
$$

Here, $\succ^{\prime}$ coincides with Grinberg's $\succ$, which we will denote by $\succ_{G}$ to avoid confusions.

Lemma 4.1. For any two words $u$ and $v$,

$$
u \prec^{\prime} v=\sum_{v_{1} v_{2}=v}(-1)^{\left|v_{1}\right|}\left(\overline{v_{1}} u\right) ш v_{2},
$$

where $\bar{w}$ denotes the mirror image of a word $w$.

Proof. By definition

$$
a u \prec^{\prime} b v=a u ш b v-a u \succ^{\prime} b v,
$$

and since

$$
a u \succ^{\prime} b v=b a u \prec^{\prime} v,
$$

the result follows by induction.

For example,

(90) $1234 \prec^{\prime} 567=1(234 ш 567)=1234$ ш $567-51234 ш 67+651234$ ш 7 - 7651234.

In FQSym, this implies

$$
\mathbf{F}_{\sigma} \prec^{\prime} \mathbf{F}_{\tau}=\sum_{u v=\tau[k]}(-1)^{|u|} \mathbf{F}_{(\bar{u} \cdot \sigma) ш v} .
$$

For example,

$$
\mathbf{F}_{2143} \prec^{\prime} \mathbf{F}_{312}=\mathbf{F}_{2143 ш 756}-\mathbf{F}_{72143 ш 56}+\mathbf{F}_{572143 ш 6}-\mathbf{F}_{6572143} .
$$

4.2. Descents in half-Shuffles. For $w \in A^{*}$, let $\operatorname{alph}(w) \subseteq A$ be the set of letters occuring in $w$. The following property appears as Lemma 4.1 in [30]:

LEMMA 4.2. If $\operatorname{alph}(u) \cap \operatorname{alph}(v)=\varnothing$, then

$$
\langle u ш v\rangle=\langle u\rangle\langle v\rangle,
$$

where the linear map \langle\rangle is defined by $\langle u\rangle:=F_{C(u)}$, where $C(u)$ denotes the descent composition of the word $u$. In particular, the descents of the elements of a shuffle on disjoint alphabets depend only on the descents of the initial elements.

There is a refined statement for the dendriform half-products [30, Thm. 4.2] that we adapt to our half-products: 
THEOREM 4.3. Let $u=u_{1} \cdots u_{k}$ and $v=v_{1} \cdots v_{\ell}$ be two nonempty words of respective lengths $k$ and $\ell$. If $\operatorname{alph}(u) \cap \operatorname{alph}(v)=\varnothing$, then

$$
\left\langle u \prec^{\prime} v\right\rangle=\left\langle\sigma \prec^{\prime} \tau\right\rangle
$$

where $\sigma=\operatorname{std}(u)$ and $\tau=\operatorname{std}(v)[k]$ if $u_{1}<v_{1}$, and $\sigma=\operatorname{std}(u)[\ell]$ and $\tau=\operatorname{std}(v)$ if $u_{1}>v_{1}$.

For example, we have

$$
\begin{aligned}
& \left\langle 14 \prec^{\prime} 23\right\rangle=\langle 1423+1243+1234\rangle=F_{22}+F_{31}+F_{4} \\
& \left\langle 12 \prec^{\prime} 34\right\rangle=\langle 1234+1324+1342\rangle=F_{4}+F_{22}+F_{31},
\end{aligned}
$$

whereas

$$
\begin{aligned}
& \left\langle 24 \prec^{\prime} 13\right\rangle=\langle 2413+2143+2134\rangle=F_{22}+F_{121}+F_{13} \\
& \left\langle 34 \prec^{\prime} 12\right\rangle=\langle 3412+3142+3124\rangle=F_{22}+F_{121}+F_{13} .
\end{aligned}
$$

4.3. Projection onto QSym. Let $\pi:$ FQSym $\rightarrow$ QSym be the canonical projection sending $\mathbf{F}_{\sigma}$ to $F_{C(\sigma)}$. Then $\pi$ is compatible with the products of both structures, and we can define half-products $\prec^{\prime}$ and $\succ^{\prime}$ on QSym in such a way that $\pi$ will be compatible with the half-products as well. This follows from an even more general trivial property. Let us consider $\sigma$ and $\sigma^{\prime}$ having the same descents and $\tau$ and $\tau^{\prime}$ also. Any word $w$ in the shifted shuffle $\sigma \in \tau=\sigma ш \tau[k]\left(\sigma \in \mathfrak{S}_{k}\right)$ is characterized by the sequence of positions of the letters of $\sigma$. Then the word $w^{\prime}$ in $\sigma^{\prime} \cup \tau^{\prime}$ with the same sequence of positions of the letters of $\sigma^{\prime}$ has obviously the same descents as $w$. Since it is true for all elements, it is in particular true when one sums over subsets of the shuffle, e.g., $\prec^{\prime}$ and $\succ^{\prime}$. Thus, if $C(\sigma)=I$ and $C(\tau)=J$, we can define in QSym

$$
F_{I} \prec^{\prime} F_{J}:=\pi\left(\mathbf{F}_{\sigma} \prec^{\prime} \mathbf{F}_{\tau}\right) \text {. }
$$

For example,

$$
\mathbf{F}_{21} \prec^{\prime} \mathbf{F}_{132}=\mathbf{F}_{21354}+\mathbf{F}_{23154}+\mathbf{F}_{23514}+\mathbf{F}_{23541}
$$

so that

$$
F_{11} \prec^{\prime} F_{21}=F_{131}+F_{221}+F_{32}+F_{311},
$$

which could have been computed as well from

$$
\mathbf{F}_{21} \prec^{\prime} \mathbf{F}_{231}=\mathbf{F}_{21453}+\mathbf{F}_{25153}+\mathbf{F}_{24513}+\mathbf{F}_{24531} \text {. }
$$

Applying Lemma 4.2, we can project (91) to QSym. If the descent composition of $u$ is $H$ and that of $\sigma$ is $I$, the descent composition of $\bar{u}$ is the conjugate composition $H^{\sim}$, and if $u_{1}>\sigma_{1}$, the descent composition of $\bar{u} \cdot \sigma$ is $H^{\sim} \cdot I$. We have therefore

$$
F_{I} \prec^{\prime} F_{J}=\sum_{J \in\{H K, H \triangleright K\}}(-1)^{|H|} F_{H \sim \cdot I} F_{K},
$$

where for two compositions $H=\left(h_{1}, \ldots, h_{r}\right)$ and $K=\left(k_{1}, \ldots, k_{s}\right)$

$$
H K=\left(h_{1}, \ldots, h_{r}, k_{1}, \ldots, k_{s}\right) \text { and } H \triangleright K=\left(h_{1}, \ldots, h_{r}+k_{1}, \ldots, k_{s}\right) .
$$

For example,

$$
\mathbf{F}_{21} \prec^{\prime} \mathbf{F}_{132}=\mathbf{F}_{21 ш 354}-\mathbf{F}_{321 ш 54}+\mathbf{F}_{5321 ш 4}-\mathbf{F}_{45321},
$$

so that

$$
\pi\left(\mathbf{F}_{21} \prec^{\prime} \mathbf{F}_{132}\right)=F_{11} F_{21}-F_{111} F_{11}+F_{1111} F_{1}-F_{2111},
$$

corresponding to the decompositions $J=\varnothing \cdot 21,1 \triangleright 11,2 \cdot 1$, and $21 \cdot \varnothing$.

Since the antipode $S$ of $Q S y m$ is given by

$$
S\left(F_{H}\right)=(-1)^{|H|} F_{H \sim},
$$


and the coproduct by

$$
\Delta\left(F_{J}\right)=\sum_{J \in\{H K, H \triangleright K\}} F_{H} \otimes F_{K}
$$

we obtain in this way [14, Detailed version (ancillary file), Thm. 3.15]:

ThEOREM 4.4. Let the $\prec^{\prime}$ product on QSym be defined by (97). Then, for $f, g \in$ QSym,

$$
f \prec^{\prime} g=\sum_{(g)}\left(S\left(g_{(1)}\right) \bullet f\right) g_{(2)}
$$

where $F_{I} \bullet F_{J}:=F_{I \cdot J}$ and $\Delta g=\sum_{(g)} g_{(1)} \otimes g_{(2)}$.

For example, applying $\pi$ to (92), we obtain

$$
F_{121} \prec^{\prime} F_{12}=F_{121} F_{12}-F_{1121} F_{2}+F_{2121} F_{1}-F_{12121}
$$

4.4. Quasi-Differential OPERATORs. Theorem 4.4 can be reformulated in terms of quasi-differential operators, as in [14]. According to [20, Def. 4.5], for $f \in$ QSym,

$$
f(X-Y)=\sum_{I} S\left(F_{I}\right)(Y) \cdot R_{I}^{\perp}(f)(X),
$$

where the quasi-differential operator $R_{I}^{\perp}$ is defined as in [14], i.e., as the adjoint of the linear map $f \mapsto R_{I} f$. Indeed, recall that $f(X-Y)$ is defined as $f((-Y) \hat{+} X)$, where $\hat{+}$ is the ordinal sum of alphabets, $f(-Y)=S(f)(Y)$. Now,

$$
\begin{aligned}
f(X \hat{+} Y) & =\sum_{J, K}\left\langle\Delta f, R_{J} \otimes R_{K}\right\rangle F_{J}(X) F_{K}(Y) \\
& =\sum_{J, K}\left\langle f, R_{J} R_{K}\right\rangle F_{J}(X) F_{K}(Y) \\
& =\sum_{J, K}\left\langle R_{J}^{\perp} f, R_{K}\right\rangle F_{J}(X) F_{K}(Y) \\
& \left.=\sum_{J} F_{J}(X) \sum_{K}\left\langle R_{J}^{\perp} f, R_{k}\right\rangle\right) F_{K}(Y) \\
& =\sum_{J} F_{J}(X)\left(R_{J}^{\perp} f\right)(Y) .
\end{aligned}
$$

Replacing $X, Y$ by $-Y, X$, this becomes

$$
f(-Y \hat{+} X)=\sum_{J} F_{J}(-Y)\left(R_{J}^{\perp} f\right)(X)=\sum_{J} S\left(F_{J}\right)(Y) \cdot R_{J}^{\perp}(f)(X),
$$

We can therefore rewrite (107) as

$$
f \prec^{\prime} g(X)=[g(X-Y) \bullet Y f(Y)]_{Y=X} .
$$

For example

$$
\begin{aligned}
F_{12}(X-Y) & =F_{12}((-Y) \hat{+} X) \\
& =F_{12}(-Y)+F_{11}(-Y) F_{1}(X)+F_{1}(-Y) F_{2}(X)+F_{12}(X) \\
& =-F_{12}(Y)+F_{2}(Y) F_{1}(X)-F_{1}(Y) F_{2}(X)+F_{12}(X) .
\end{aligned}
$$

Taking the $\bullet$ product with $F_{121}(Y)$, we obtain

$$
\begin{aligned}
F_{12}(X & -Y) \bullet F_{121}(Y) \\
& =-F_{12121}(Y)+F_{2121}(Y) F_{1}(X)-F_{1121}(Y) F_{2}(X)+F_{121}(Y) F_{12}(X),
\end{aligned}
$$

and setting $Y=X$, we recover (108). 
Similarly, we have for the right product

$$
u \succ^{\prime} v=\sum_{u_{1} u_{2}=u}(-1)^{\left|u_{1}\right|} u_{2} \amalg \overline{u_{1}} v .
$$

Thus, on $Q S y m$, if one defines $\triangleright$ by $F_{I} \triangleright F_{J}=F_{I \triangleright J}$, then

$$
f \succ^{\prime} g=\sum_{(f)}\left(S\left(f_{(1)}\right) \triangleright g\right) f_{(2)} .
$$

This is precisely [14, Thm. 3.7]. For example,

$$
\mathbf{F}_{132} \succ^{\prime} \mathbf{F}_{21}=\mathbf{F}_{132 \uplus 54-32 ш 154+2 ш 3154-23154},
$$

which projects onto

(123) $F_{21} \succ^{\prime} F_{11}=F_{21} F_{11}-F_{11} F_{21}+F_{1} F_{121}-F_{221}=F_{131}+F_{1121}+F_{122}+F_{1211}$.

4.5. StandARD DENDRIFORM STRUCTURES. With the usual definitions

$$
u a \prec v b=(u ш v b) a, \quad u a \succ v b=(u a ш v) b,
$$

the half-shuffle identity becomes

$$
u \prec v=\sum_{v_{1} v_{2}=v}(-1)^{\left|v_{2}\right|} u \overline{v_{2}} \amalg v_{1}
$$

which induces an operation on QSym defined by

$$
F_{I} \prec F_{J}=\sum_{J \in\{H K, H \triangleright K\}}(-1)^{|K|} F_{I \triangleright K \sim F_{H}},
$$

or equivalently,

$$
f \prec g=\sum_{(g)} g_{(1)}\left(f \triangleright S\left(g_{(2)}\right)\right) .
$$

For example,

$$
\mathbf{F}_{21} \prec \mathbf{F}_{132}=\mathbf{F}_{35421}+\mathbf{F}_{35241}+\mathbf{F}_{32541}+\mathbf{F}_{23541}
$$

and

$$
F_{11} \prec F_{21}=F_{2111}+F_{221}+F_{1211}+F_{311} .
$$

Then,

$$
F_{I} \prec F_{J}=\left.F_{I}(Y) \triangleright_{Y} F_{J}(X-Y)\right|_{Y=X} .
$$

For example,

$$
F_{21}(X-Y)=F_{21}(X)-F_{1}(Y) F_{2}(X)+F_{2}(Y) F_{1}(X)-F_{21}(Y)
$$

and

$$
\begin{aligned}
F_{11}(Y) \triangleright_{Y} & F_{21}(X-Y) \\
& =F_{11}(Y) F_{21}(X)-F_{12}(Y) F_{2}(X)+F_{13}(Y) F_{1}(X)-F_{131}(Y)
\end{aligned}
$$

so that

$$
F_{11} \prec F_{21}=F_{11} F_{21}-F_{12} F_{2}+F_{13} F_{1}-F_{131} .
$$

For the right product, we have

$$
u \succ v=\sum_{u_{1} u_{2}=u}(-1)^{\left|u_{2}\right|} u_{1} ш v \overline{u_{2}},
$$

and on QSym,

$$
f \succ g=\sum_{(f)} f_{(1)}\left(g \bullet S\left(f_{(2)}\right)\right) .
$$


For example,

$$
\mathbf{F}_{21} \succ \mathbf{F}_{231}=\mathbf{F}_{21 \uplus 453-2 \uplus 4531+45312}
$$

which yields on QSym

(137) $F_{11} \succ F_{21}=F_{11} F_{21}-F_{1} F_{211}+F_{212}=F_{131}+F_{122}+F_{221}+F_{32}+F_{1121}+F_{212}$.

4.6. GrinberG's operations. In [14], a left product $\prec_{G}$ on $Q S y m$ is induced from an operation on monomials. This operation is the commutative image of the left tridendriform product on words defined in [28] (with the use of min instead of max, so that we shall consistently denote it by $\prec^{\prime}$ ), and amounts to taking the canonical projection $\pi$ : WQSym $\rightarrow$ QSym of the tridendriform product of WQSym:

$$
M_{I} \prec_{G} M_{J}:=\pi\left(\mathbf{M}_{u} \prec^{\prime} \mathbf{M}_{v}\right)
$$

where $u, v$ are any packed words of evaluation $I, J$.

For example, to evaluate $F_{11} \prec_{G} F_{21}$, we compute $M_{11} \prec_{G}\left(M_{21}+M_{111}\right)$, hence $\mathbf{M}_{21} \prec^{\prime}\left(\mathbf{M}_{132}+\mathbf{M}_{121}\right)$ :

$$
\begin{gathered}
\mathbf{M}_{21} \prec^{\prime} \mathbf{M}_{132}=\mathbf{M}_{21243}+\mathbf{M}_{21354}+\mathbf{M}_{31243}+\mathbf{M}_{41243}+\mathbf{M}_{51243}+\mathbf{M}_{41253}+\mathbf{M}_{31254}, \\
\mathbf{M}_{21} \prec^{\prime} \mathbf{M}_{121}=\mathbf{M}_{21232}+\mathbf{M}_{31212}+\mathbf{M}_{21343}+\mathbf{M}_{31242}+\mathbf{M}_{41232}
\end{gathered}
$$

so that

$$
\begin{aligned}
F_{11} \prec_{G} F_{21} & =3 M_{1211}+2 M_{1121}+M_{1112}+M_{122}+M_{131}+4 M_{1111} \\
& =F_{131}+F_{122}+F_{1121}+F_{1211},
\end{aligned}
$$

which is different from all previous examples such as (99).

This operation can then be lifted to FQSym as

$$
\mathbf{F}_{\sigma} \prec_{G} \mathbf{F}_{\tau}:=\mathbf{F}_{\sigma[l] \prec^{\prime} \tau},
$$

where $l=|\tau|$, and $a u \prec^{\prime} b v$ is defined as $a(u \uplus b v)$. Then Equation (87) yields [14, Thm. 3.7]

$$
F_{I} \prec_{G} F_{J}=\sum_{J \in\{H K, H \triangleright K\}}(-1)^{|H|} F_{H \sim I} F_{K}
$$

For example,

$$
\mathbf{F}_{21} \prec_{G} \mathbf{F}_{132}=\mathbf{F}_{54132}+\mathbf{F}_{51432}+\mathbf{F}_{51342}+\mathbf{F}_{51234},
$$

and one can check that

$$
54 \prec^{\prime} 132=54 \text { ш } 132-154 \text { ш } 32+3154 \text { ш } 2-23154,
$$

so that

$$
F_{11} \prec_{G} F_{21}=F_{11} F_{21}-F_{21} F_{11}+F_{121} F_{1}-F_{221} .
$$

Alternatively, we can describe $\prec_{G}$ as

$$
\mathbf{F}_{\sigma} \prec_{G} \mathbf{F}_{\tau}=\mathbf{F}_{\tau} \succ^{\prime} \mathbf{F}_{\sigma} .
$$

On QSym, this translates as

$$
f \prec_{G} g=\sum_{(g)} g_{(2)}\left(S\left(g_{(1)}\right) \triangleright f\right)
$$

which is now [14, Thm. 3.7] in its original form.

Grinberg's expression of the dual immaculate basis can now be restated as

$$
\mathfrak{S}_{I}^{*}=\left(\cdots\left(F_{i_{r}} \succ^{\prime} F_{i_{r-1}}\right) \succ^{\prime} \cdots\right) \succ^{\prime} F_{i_{1}} .
$$


For example,

$$
\mathfrak{S}_{221}^{*}=\left(F_{1} \succ^{\prime} F_{2}\right) \succ^{\prime} F_{2}=\left(F_{12}+F_{21}\right) \succ^{\prime} F_{2} .
$$

Note 4.5. On FQSym, the operation $\prec_{G}$ is not a left dendriform product in the usual sense, as it is in fact a flipped right product. The $\prec^{\prime}$ operation on WQSym which induces it does not preserve the standard FQSym subalgebra of WQSym.

Finally, it is also possible to deduce Theorem 3.6 from Grinberg's results. The following argument has been suggested by an anonymous referee.

Let $f \mapsto \bar{f}$ be the anti-involution of $\mathbf{F Q S y m}$ defined by $\overline{\mathbf{F}_{\sigma}}=\mathbf{F}_{\omega \sigma \omega}$, and denote by $\pi:$ FQSym $\rightarrow$ QSym the canonical projection $\pi\left(\mathbf{F}_{\sigma}\right)=F_{C(\sigma)}$. As already observed, $\pi(\bar{f})=\overline{\pi(f)}$. It is easy to see that

$$
\overline{a \prec b}=\bar{b} \succ_{G} \bar{a} \text { for all } a, b \in \mathbf{F Q S y m} .
$$

Moreover, in $[14, \S 6]$, it is said that

$$
\pi(a) \prec_{G} \pi(b)=\pi(a \succ b) \quad \text { for all } a, b \in \mathbf{F Q S y m} .
$$

Now, let $I=\left(i_{1}, i_{2}, \ldots, i_{r}\right)$ be a composition. Then, [14, Cor. 4.7] shows that

$$
\begin{aligned}
& \mathfrak{S}_{I}^{*}=h_{i_{1}} \prec_{G}\left(h_{i_{2}} \prec_{G}\left(\cdots\left(h_{i_{r}} \prec_{G} 1\right) \cdots\right)\right) \\
& \quad=h_{i_{1}} \prec_{G}\left(h_{i_{2}} \prec_{G}\left(\cdots\left(h_{i_{r-1}} \prec_{G} h_{i_{r}}\right) \cdots\right)\right) \\
& \left.\quad=\pi\left(S_{i_{1}}\right) \prec_{G}\left(\pi\left(S_{i_{2}}\right) \prec_{G}\left(\cdots \prec_{G} \pi\left(S_{i_{r-1}}\right) \prec_{G} \pi\left(S_{i_{r}}\right)\right) \cdots\right)\right) \\
& \text { (since } \left.h_{k}=\pi\left(S_{k}\right) \text { for all } k\right) \\
& \quad=\pi\left(\left(\left(\cdots\left(S_{i_{r}} \succ_{G} S_{i_{r-1}}\right) \succ_{G} \cdots\right) \succ_{G} S_{i_{2}}\right) \succ_{G} S_{i_{1}}\right)
\end{aligned}
$$

(by applying (150) many times)

$$
\left.=\pi\left(\left(\left(\cdots \overline{\left(\overline{i_{r}}\right.} \succ_{G} \overline{S_{i_{r-1}}}\right) \succ_{G} \cdots\right) \succ_{G} \overline{S_{i_{2}}}\right) \succ_{G} \overline{S_{i_{1}}}\right)
$$

(since $\overline{S_{k}}=S_{k}$ )

$$
=\pi\left(\overline{S_{i_{1}} \prec\left(S_{i_{2}} \prec\left(\cdots \prec\left(S_{i_{r-1}} \prec S_{i_{r}}\right) \cdots\right)\right)}\right)
$$

(by applying (149) many times)

$$
\begin{aligned}
& =\pi\left(\overline{C_{I}(A)}\right) \quad \text { as seen in the proof of Lemma } 3.1 \\
& =\overline{\pi\left(C_{I}(A)\right)} \\
& =\overline{C_{I}(X)} .
\end{aligned}
$$

Hence, Theorem 3.6 follows from [14, Cor. 4.7].

\section{Hopf Algebras of SET PARTitions}

We have seen that the Bell polynomial $\boldsymbol{B}_{n}$ can be identified with the formal sum of permutations avoiding $21-3$ (up to the $Y^{I}$ which can be reconstructed from the descent sets). This raises the question of the existence of a Hopf subalgebra or quotient of FQSym whose bases are naturally labeled by these permutations.

The most obvious Hopf algebra of set partitions is WSym, or symmetric functions in noncommuting variables (not to be confused with noncommutative symmetric functions Sym). In [17], a quotient of WSym isomorphic to Sym and a QSym subalgebra of its dual are related to Bell polynomials. In [5], analogues of the Bell polynomials in various other Hopf algebras are considered. 
5.1. The Bell Hopf Algebra. The Hopf algebra WSym is cocommutative. Quite often, combinatorial objects also admit a self-dual Hopf algebra structure. Such an algebra has been constructed by Rey [34, 35] for set partitions from the BursteinLankham correspondence, a combinatorial construction derived from the patience sorting algorithm. More precisely, the Bell classes of Rey are indexed by permutations avoiding $23-1$, and these are their minimal elements (for the weak order). We can modify the construction so as to have classes whose maximal elements avoid $21-3$ as follows.

Let $A$ be a totally ordered alphabet. The (modified) Bell congruence on $A^{*}$ is generated by the relations ${ }^{(4)}$

$$
b u c a \equiv b u a c \quad \text { if } a<b<c \text { and all letters of } u \text { are smaller than } b .
$$

This is a refinement of the reverse sylvester congruence, which is defined by the same relations without restriction on $u$.

As for the sylvester or reverse sylvester congruences, we have:

THEOREM 5.1. The Bell equivalence classes of permutations, (i.e., equivalence classes of words with no equal letters under the Bell congruence) are intervals of the right weak order on the symmetric group. These intervals consist of the linear extensions of posets which will be explicitly described below, and the maximal elements of these intervals are the 21 - 3-avoiding permutations.

Identifying set partitions $\pi$ with their representatives $\hat{\pi}^{b}$ as 21 - 3 -avoiding permutations, we set

$$
P_{\pi}=\sum_{\sigma \equiv \pi} \mathbf{F}_{\sigma}
$$

TheOrem 5.2 (Rey [35]). The $P_{\pi}$ span a Hopf subalgebra of FQSym.

5.2. The Poset of Set PARtitions. Since the long version of Rey's paper [35] has never been published, we shall provide detailed proofs of his results (in our modified version), to which we add the explicit description of the posets.

5.2.1. From permutations to set partitions. Let us first define an insertion algorithm. This is a rewriting of the Patience Sorting Algorithm defined by Burstein and Lankham adapted to our setting, which amounts to applying some trivial involutions on words. Precisely, if we invert the total order of the alphabet, our algorithm becomes Algorithm 3.1 of [34], our blocks being Rey's piles read downwards.

The first object that we create is a set partition, that we shall regard as ordered in such a way that the maximal elements of the blocks decrease from left to right.

Let $w=w_{1} \ldots w_{n}$ be a word with no repeated letters over a totally ordered alphabet. Put $S=\varnothing$. Then, read $w$ from left to right and for each letter $w_{i}$ do

- Step 1: Let $s$ be the block of $S$ whose maximal element is smaller than (or equal to) $w_{i}$ and is greater than all other maximal elements smaller than $w_{i}$.

- Step 2: If $s$ does not exist, add $\left\{w_{i}\right\}$ to $S$. If $s$ exists, insert $w_{i}$ into it.

The result of this algorithm will be denoted by $\operatorname{PSA}(w)$.

We shall display the blocks of $S$ as columns, increasing from top to bottom (so that an element is inserted at the bottom of its column), the columns being ordered from left to right with their maximal elements in decreasing order.

\footnotetext{
${ }^{(4)}$ As observed by Grinberg (private communication), an alternative description is $b c u \equiv b u c$ for $b<c$ if all letters of $u$ are $<b$.
} 
For example, the insertion of $(3,1,2,6,4,5,7)$ follows the steps:

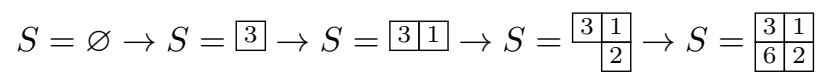

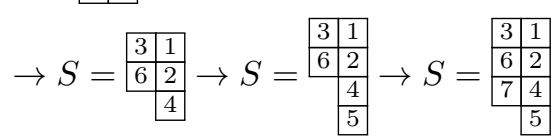

5.2.2. From set partitions to posets. Now, starting from a set partition $S$, we shall build a poset $P$ on the ground set $\{1,2, \ldots, n\}$ whose relation $>_{P}$ is generated by the following requirements: order $S$ as above, and, for any element $x$ of a block $s$ of $S$, write $x>_{P} x^{\prime}$ where $x^{\prime}$ is the greatest element smaller than $x$ in $s$ and write $x>_{P} x^{\prime \prime}$ where $x^{\prime \prime}$ is the smallest element greater than $x$ in the block immediately to the left of $s$ in $S$ (if such a block and such an element exist). The transitive closure of $>_{P}$ defines a poset which will be denoted by $P(S)$ or by $P(w)$ if $S$ is the result of the (modified) patience sorting algorithm applied to $w$. Note that by removing the edges of the Hasse diagram of $P(S)$ where the element above is greater than the element below, one recovers $S$ itself. Hence it makes sense to refer to the columns of $S$ as the corresponding columns of the poset $P(S)$. For $w$ in $P$, we shall write $C(w)$ for its column.

As usual with posets, we shall only represent the covering relations. For example, starting with the set $S$ computed before, we get (orienting the Hasse diagrams upsidedown, with minimal elements at the top):

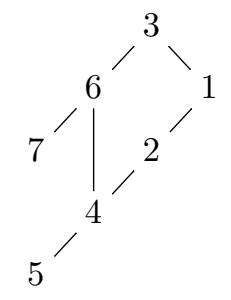

Indeed, the first column of $S$ is $(3,6,7)$ and $6>3$ and $7>6$. Now, on the second column we have $5>4>2>1$ and the relations between these elements and the first column are: $1>_{P} 3,2>_{P} 3,4>_{P} 6$, and $5>_{P} 6$, whence the Hasse diagram above.

Starting with $S=(6,10,11|2,4,8,9| 3,7|5| 1)$, one gets

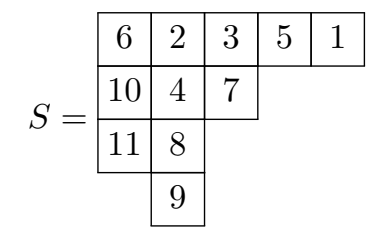

and

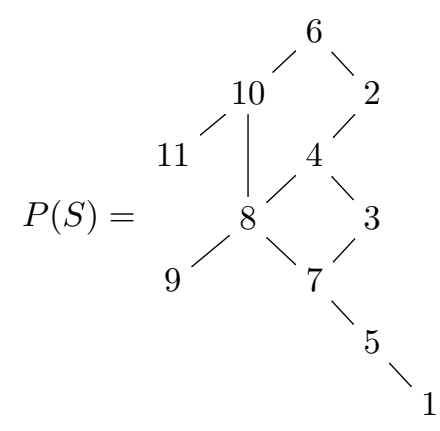


Note that in this representation, the columns of $P(S)$ are the straight lines going from south-west to north-east.

5.3. Proofs. We shall now prove Theorem 5.1: all elements in a given Bell class have the same poset $P(w)=P(\operatorname{PSA}(w)$ ), and the linear extensions (taken from top to bottom in our representation) of such a poset give elements of the same Bell class. Hence being in the same Bell class is equivalent to having the same poset.

5.3.1. All elements in a given Bell class have the same poset. First, let us prove that all elements in a given Bell class have the same poset, or, equivalently, the same result by the PSA algorithm. We only need to prove this for two elements $w$ and $w^{\prime}$ obtained from one another by a single rewriting rule. So, let us consider $w=v b u a c$ and $w^{\prime}=v b u c a$ where all letters of $u$ are smaller than $b$. When $b$ is inserted, it is the bottommost element of its column, and it remains so during the insertion of all $u$, since all the letters of $u$ are smaller than $b$. Now, a letter $c>b$ is necessarily inserted into a column to the left of $C(b)$ or into it, independently of the insertion of $a$. Meanwhile, a letter $a$ smaller than $b$ is necessarily inserted into a column strictly to the right of $C(b)$ (whether or not $c$ has already been inserted). Thus, the insertions of $c$ and $a$ do not interfere with each other. So $w$ and $w^{\prime} \operatorname{satisfy} \operatorname{PSA}(w)=\operatorname{PSA}\left(w^{\prime}\right)$.

Note that the condition that all letters of $u$ are smaller than $b$ is necessary to ensure that $c$ and $a$ cannot both be inserted in the same column, which would prevent $w$ and $w^{\prime}$ from yielding the same result by the modified patience sorting algorithm.

5.3.2. All linear extensions of a poset are Bell congruent. Let us now prove that all linear extensions of a poset $P(\sigma)$ are Bell congruent to $\sigma .^{(5)}$ First note that the poset obtained from a permutation $\sigma$ has a unique minimal element $m$ at its top: indeed, it is easy to see that whenever $p<q$, each element of the $q$ th column is $>_{P}$ to the smallest element of the $p$ th column. Now, $\sigma$ is a linear extension of it, since when we insert a new letter $r$, we do not get a new covering relation of the form $s>_{P} r$.

One can thus rebuild the corresponding set partition $\operatorname{PSA}(\sigma)$ since each column of $P(\sigma)$ is a saturated chain of the poset $P(\sigma)$, and conversely, if we remove all edges of the form $u>_{P} v$ satisfying $u<v$ from the Hasse diagram of $P(\sigma)$, then only these saturated chains remain. Let us define a Bell poset as a poset $P(S)$ for a set partition $S$ of some finite set of integers, not necessarily $\{1,2, \ldots, n\}$. It is easy to see that removing a maximal element from a Bell poset yields a Bell poset, which implies that any down-closed subset of a Bell poset is again a Bell poset. So, let us now consider two values $a<c$, both maximal elements of $P$, and let $P^{\prime}$ be the poset obtained from $P$ by removing $a$ and $c$. By induction on $n$, we can assume that all linear extensions of $P^{\prime}$ are Bell-congruent with one another. To prove that the same holds for $P$, we only need to prove that there exists one linear extension $v$ of $P^{\prime}$ such that $v \cdot a c \equiv v \cdot c a$ : indeed, all linear extensions of a poset can be reached from one another by such exchanges.

First, note that $C(a)$ is to the right of $C(c)$ since they are both maximal elements of their column. Let us prove that there exists a linear extension of $P^{\prime}$ that ends with a letter $b$ such that $a<b<c$, possibly followed by letters smaller than $a$. We shall use twice a very simple observation: if $x$ is the last letter of a column, then there are linear extensions of the associated poset where $x$ is only followed by letters smaller than itself. Indeed, the letters that must appear after $x$ in all linear extensions of the poset are letters belonging to columns to the right of $C(x)$, hence all smaller than $x$.

Now, if $C(c)$ is not the column immediately to the left of $C(a)$, define $b$ as the maximal element of this column. It satisfies $a<b<c$ and all elements after it in

${ }^{(5)}$ Grinberg (private communication) has proposed another proof, an induction of the length of $\sigma$. 
the linear extensions of $P^{\prime}$ are smaller than $b$, and in fact smaller than $a$. So there are linear extensions of $P^{\prime}$ where the rewriting $w c a \equiv w a c$ is possible. Otherwise, since $a$ and $c$ are both maximal elements of $P$, they cannot be comparable. Define $b$ as the letter immediately above $c$ in its column. It exists since otherwise $a$ would be connected to $c$ (definition of the posets from the sets of columns). Now, $b$ might not be a maximal element of $P^{\prime}$ but in any case, the values that are below $b$ in $P^{\prime}$ are strictly to its right in the partition, hence are all smaller than $a$, again. So there are linear extensions of $P^{\prime}$ ending with $b$ and then letters smaller than $a$. So in both cases, we found a value $b$ allowing to rewrite one linear extension $w$ of $P^{\prime}$ concatenated to $a c$ with $w \cdot c a$, thus proving that all linear extensions of a poset $P(\sigma)$ are Bell congruent to $\sigma$.

5.3.3. Our posets are regular. Now that we have obtained the equivalence between Bell classes and the linear extensions of our posets, it only remains to prove that these posets (regarded as labeled posets in an obvious way) are regular to conclude that the classes are intervals of the weak order [4, Thm. 5.8]. We shall make use of a very simple property: if a value $u$ belongs to a column to the left of a value $v$, and if $u<v$ then $u<_{P} v$. Indeed, consider the column $C^{\prime}$ immediately to the left of $C(v)$. Then $v$ is $>_{P}$ the smallest value $v^{\prime}$ greater than itself in $C^{\prime}$, hence below all values smaller than itself. Now the same property holds for $v^{\prime}>v$ on the next column to its left and so on.

Let us now consider two elements $x$ and $z$ such that $x<_{P} z$, so that $C(x)$ is equal to, or to the left of $C(z)$, which we shall write as $C(x) \preceq C(z)$.

Two cases may appear: $x<z$ or $x>z$. Let us consider the first case and let $y$ be another element of $P$ such that $x<y<z$. In that case, either $C(y) \preceq C(z)$ or $C(y) \succeq C(x)$. In both cases, apply our previous result and conclude that either $x>_{P} y$ or $y<_{P} z$. Let us now consider the second case where $x>z$ and let $y$ be another element of $P$ such that $x>y>z$. If $C(y) \preceq C(z)$ or $C(y) \succeq C(x)$, the same argument as before applies. Otherwise, it means that $C(x) \preceq C(y) \preceq C(z)$. But in that case, since there is a saturated chain from $x$ down to $z$, all elements in between are either comparable to $x$ (if they are below the path) or to $z$ (if they are above the path). So in all cases, we proved that $y$ satisfies either $x<_{P} y$ or $y<_{P} z$, hence proving that our posets are regular.

5.3.4. The maximal elements of the Bell classes avoid 21 - 3. Let us finally prove that the maximal elements of the Bell classes avoid the pattern $21-3$. There are several ways to prove this. One could say for example that the Bell classes are in bijection with set partitions, hence splitting the permutations of size $n$ into as many classes as the number of permutations avoiding $21-3$. Now, if a permutation $\sigma$ does not avoid $21-3$, let us consider such a pattern with letters $b a \ldots c$ in $\sigma$ where the distance between $a$ and $c$ is minimal. Then the letter immediately to the left of $c$ (if it exists: the other case is easy) has to be smaller than $b$, hence denoted naturally by $a^{\prime}$. All letters $x$ between $b$ and $a^{\prime}$ are necessarily smaller than $b$, otherwise $b a \cdots x \cdots a^{\prime} c$ would have $b a \cdot x$ as a shorter pattern $21-3$, which would contradict the minimality of the initial one. So we are in the conditions of the Bell congruence, and $\sigma$ is not a maximal element of its class. So all maximal elements necessarily avoid the pattern $21-3$. Since both sets, the maximal elements of Bell classes and the permutations avoiding $21-3$ have same cardinality, they must be equal.

Another proof consists in applying the greedy algorithm that takes a Bell poset as entry and takes the linear extension where at each step one chooses the maximal available value. It is easy to see that these elements necessarily avoid the pattern $21-3$ and conclude in the same way as before. 
Note that the minimal elements are not characterized by pattern avoidance.

5.3.5. Proof of Theorem 5.2. As for Theorem 5.2, it follows from a well-known result about algebras defined by congruences, see e.g., Theorem 2.1 of [32] or Chapter 2.1 of [33].

Acknowledgements. This research has been partially supported by the ANR program CARMA. Thanks also to Darij Grinberg for his useful comments on the first version of this text.

\section{REFERENCES}

[1] Andrew Michael Baxter, Algorithms for permutation statistics, ProQuest LLC, Ann Arbor, MI, 2011, Thesis (Ph.D.)-Rutgers The State University of New Jersey - New Brunswick (USA).

[2] Chris Berg, Nantel Bergeron, Franco Saliola, Luis Serrano, and Mike Zabrocki, A lift of the Schur and Hall-Littlewood bases to non-commutative symmetric functions, Canad. J. Math. 66 (2014), no. 3, 525-565.

[3] - Indecomposable modules for the dual immaculate basis of quasi-symmetric functions, Proc. Am. Math. Soc. 143 (2015), no. 3, 991-1000.

[4] Anders Björner and Michelle L. Wachs, Permutation statistics and linear extensions of posets, J. Combin. Theory Ser. A 58 (1991), no. 1, 85-114.

[5] Ali Chouria and Jean-Gabriel Luque, $r$-Bell polynomials in combinatorial Hopf algebras, C. R. Math. Acad. Sci. Paris 355 (2017), no. 3, 243-247.

[6] Anders Claesson, Generalized pattern avoidance, Eur. J. Comb. 22 (2001), no. 7, 961-971.

[7] Jacques Désarménien, Fonctions symétriques associées à des suites classiques de nombres, Ann. Sci. Éc. Norm. Supér. 16 (1983), no. 2, 271-304.

[8] Gérard Duchamp, Florent Hivert, and Jean-Yves Thibon, Noncommutative symmetric functions. VI. Free quasi-symmetric functions and related algebras, Int. J. Algebra Comput. 12 (2002), no. 5, 671-717.

[9] Kurusch Ebrahimi-Fard, Alexander Lundervold, and Dominique Manchon, Noncommutative Bell polynomials, quasideterminants and incidence Hopf algebras, Int. J. Algebra Comput. 24 (2014), no. 5, 671-705.

[10] H. O. Foulkes, Tangent and secant numbers and representations of symmetric groups, Discrete Math. 15 (1976), no. 4, 311-324.

[11] __ Eulerian numbers, Newcomb's problem and representations of symmetric groups, Discrete Math. 30 (1980), no. 1, 3-49.

[12] Israel M. Gelfand, Daniel Krob, Alain Lascoux, Bernard Leclerc, Vladimir S. Retakh, and JeanYves Thibon, Noncommutative symmetric functions, Adv. Math. 112 (1995), no. 2, 218-348.

[13] Ira M. Gessel and Christophe Reutenauer, Counting permutations with given cycle structure and descent set, J. Combin. Theory Ser. A 64 (1993), no. 2, 189-215.

[14] Darij Grinberg, Dual creation operators and a dendriform algebra structure on the quasisymmetric functions, Canad. J. Math. 69 (2017), no. 1, 21-53.

[15] Florent Hivert, Jean-Gabriel Luque, Jean-Christophe Novelli, and Jean-Yves Thibon, The (1 $\mathbb{E})$-transform in combinatorial Hopf algebras, J. Algebr. Comb. 33 (2011), no. 2, 277-312.

[16] Florent Hivert, Jean-Christophe Novelli, and Jean-Yves Thibon, The algebra of binary search trees, Theoret. Comput. Sci. 339 (2005), no. 1, 129-165.

[17] _ Commutative combinatorial Hopf algebras, J. Algebr. Comb. 28 (2008), no. 1, 65-95.

[18] - Trees, functional equations, and combinatorial Hopf algebras, Eur. J. Comb. 29 (2008), no. $7,1682-1695$.

[19] Matthieu Josuat-Vergès, Jean-Christophe Novelli, and Jean-Yves Thibon, The algebraic combinatorics of snakes, J. Combin. Theory Ser. A 119 (2012), no. 8, 1613-1638.

[20] Daniel Krob, Bernard Leclerc, and Jean-Yves Thibon, Noncommutative symmetric functions. II. Transformations of alphabets, Int. J. Algebra Comput. 7 (1997), no. 2, 181-264.

[21] Daniel Krob and Jean-Yves Thibon, Noncommutative symmetric functions. IV. Quantum linear groups and Hecke algebras at $q=0$, J. Algebr. Comb. 6 (1997), no. 4, 339-376.

[22] Jean-Louis Loday and María O. Ronco, Hopf algebra of the planar binary trees, Adv. Math. 139 (1998), no. 2, 293-309.

[23] $\ldots$, Order structure on the algebra of permutations and of planar binary trees, J. Algebr. Comb. 15 (2002), no. 3, 253-270.

[24] Ian G. Macdonald, Symmetric functions and Hall polynomials, 2nd ed., Oxford Classic Texts in the Physical Sciences, Clarendon Press, 2015. 
[25] Clauda Malvenuto and Christophe Reutenauer, Duality between quasi-symmetric functions and the Solomon descent algebra, J. Algebra 177 (1995), no. 3, 967-982.

[26] Jean-Christophe Novelli and Jean-Yves Thibon, Construction de trigèbres dendriformes, C. R. Math. Acad. Sci. Paris 342 (2006), no. 6, 365-369.

[27] _ Polynomial realizations of some trialgebras, 18th Formal Power Series and Algebraic Combinatorics (FPSAC'06), no. 1, 2006, pp. 243-254, https://hal-upec-upem. archives-ouvertes.fr/hal-00622743.

[28] _ Hopf algebras and dendriform structures arising from parking functions, Fundam. Math. 193 (2007), no. 3, 189-241.

[29] _ Noncommutative symmetric functions and Lagrange inversion, Adv. Appl. Math. 40 (2008), no. 1, 8-35.

[30] Superization and $(q, t)$-specialization in combinatorial Hopf algebras, Electron. J. Comb. 16 (2009), no. 2, Research Paper 21 (46 pages), http://www.combinatorics.org/volume_16/ abstracts/v16i2r21.html.

[31] _ Binary shuffle bases for quasi-symmetric functions, Ramanujan J. 40 (2016), no. 1, $207-225$.

[32] _ Hopf Algebras of m-permutations, $(m+1)$-ary trees, and m-parking functions, https: //hal.archives-ouvertes.fr/hal-01289784, 2016.

[33] Janvier Nzeutchap, Correspondances de Schensted-Fomin algébres de Hopf et graphes gradués en dualité, Ph.D. thesis, Université de Rouen (France), 2008, http://www.theses.fr/ 2008ROUES046, sous la direction de Florent Hivert, 1 vol. (150 p.).

[34] Maxime Rey, A self-dual Hopf algebra on set partitions, http://igm.univ-mlv.fr/ rey/ articles/hopf_set.pdf, 2007.

[35] _ Algebraic constructions on set partitions, 19th Formal Power Series and Algebraic Combinatorics (FPSAC'07), https://hal.archives-ouvertes.fr/hal-00622741, 2007.

[36] Rainer Schimming and Saad Zagloul Rida, Noncommutative Bell polynomials, Int. J. Algebra Comput. 6 (1996), no. 5, 635-644.

[37] N. J. A. Sloane, The on-line encyclopedia of integer sequences, 2010, http://oeis.org.

[38] Jean-Yves Thibon, Lectures on noncommutative symmetric functions, in Interaction of combinatorics and representation theory, MSJ Mem., vol. 11, Mathematical Society of Japan, 2001 pp. 39-94.

Jean-Christophe Novelli, Laboratoire d'informatique Gaspard-Monge, Université Paris-Est Marne-la-Vallée, 5, Boulevard Descartes, Champs-sur-Marne, 77454 Marne-la-Vallée cedex 2, France

E-mail : novelli@u-pem.fr

Jean-Yves Thibon, Laboratoire d'informatique Gaspard-Monge, Université Paris-Est Marne-laVallée, 5, Boulevard Descartes, Champs-sur-Marne, 77454 Marne-la-Vallée cedex 2, France E-mail : jyt@u-pem.fr

FréDÉRIC Toumazet, Laboratoire d'informatique Gaspard-Monge, Université Paris-Est Marne-laVallée, 5, Boulevard Descartes, Champs-sur-Marne, 77454 Marne-la-Vallée cedex 2, France E-mail : frederic.toumazet@u-pem.fr 